CLINICAL ALERT

\title{
Encephalopathy after persistent vomiting: Three cases of non-alcohol-related Wernicke's encephalopathy
}

\author{
K Antel, N Singh, B Chisholm, J M Heckmann \\ Katherine Antel, MB ChB, and Nevadna Singh, MB ChB, are both based in the Department of Medicine, Faculty of Health Sciences, University of \\ Cape Town, South Africa. Briony Chisholm is an Information Pharmacist at the Medicines Information Centre in the Division of Pharmacology in \\ the same department, and Jeannine M Heckmann is an Associate Professor in the Division of Neurology, Department of Medicine, Groote Schuur \\ Hospital and Faculty of Health Sciences, University of Cape Town.
}

Corresponding author: J M Heckmann (jeanine.heckmann@uct.ac.za)

Wernicke's encephalopathy (WE) is a medical emergency. Although WE is commonly viewed in the context of alcoholism, it can be caused by thiamine deficiency secondary to persistent vomiting. Non-alcohol-related WE may be more catastrophic in onset and less likely to present with the classic features than WE with alcoholism as a cause. We describe three cases of WE due to persistent vomiting without alcoholism in patients with hyperemesis gravidarum, drug-induced hyperlactataemia, and an acute gastrointestinal illness in an already malnourished individual. Our cases highlight the importance of recognising WE when undernutrition, which may be caused by gastrointestinal disease or surgery, or malignancy, is compounded by vomiting. Expert guidelines suggest that WE must be considered in the emergency room in any individual with disturbed consciousness of unknown cause. Treatment is with parenteral thiamine before glucose administration.

S Afr Med J 2015;105(6):442-443. DOI:10.7196/SAMJ.9299

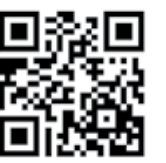

Wernicke's encephalopathy (WE) is an acute neurological condition caused by thiamine deficiency. Classically, patients with WE are thought to present with eye signs, ataxia and confusion. However, only $20 \%$ in fact have this triad of symptoms. In addition, non-alcohol-related WE is more likely to present acutely and with atypical features than WE with alcoholism as a cause. ${ }^{[1]}$ In the absence of a point-of-care diagnostic test, WE needs to be considered and pre-emptively treated in individuals at risk to avoid catastrophic consequences. ${ }^{[1,2]}$ We describe three cases encountered over 3 years at Groote Schuur Hospital, Cape Town, South Africa, to highlight the importance of treating any individual with disturbed consciousness of unknown cause with parenteral thiamine before glucose is given. ${ }^{[1,3]}$

\section{Case reports}

\section{Case 1}

A 16-year-old scholar presented to a community hospital with a 2-day history of confusion and paraparesis. She had been vomiting daily owing to a concealed 17 -week pregnancy. There was no history of alcohol or drug use.

The admitting doctors noted that she had confusion, nystagmus, ataxic arms and paraparesis. She was given intravenous fluids containing glucose and transferred to our institution within 3 days for brain magnetic resonance imaging (MRI). We found her to be agitated and confused. She was tachypnoeic and tachycardic, but her blood pressure and the findings on cardiac and chest examination were normal. She had gaze-evoked nystagmus, past-pointing in the arms, and areflexic, flaccid, paralysed legs. She was unable to sit owing to truncal ataxia.

The results of laboratory investigations were normal, including renal and thyroid function, electrolytes and vitamin $B_{12}$ levels; transaminase enzymes were only mildly elevated. The cerebrospinal fluid (CSF) was normal. A chest radiograph showed borderline cardiomegaly, an electrocardiogram demonstrated diffuse T-wave inversion inferolaterally, and an echocardiogram was normal.
Nerve conduction studies were consistent with a sensorimotor axonopathy. Brain computed tomography (CT) showed symmetrical hypodensities in the caudate nuclei and magnetic resonance imaging (MRI) showed an increased signal on T2-weighted/fluid attenuation inversion recovery (FLAIR) sequences in the same region, although extending to involve the anterior lentiform nuclei (Fig. 1).

A clinical diagnosis of WE with beriberi was made after $\sim 6$ days of symptoms. Intravenous (IV) thiamine was started (300 mg 8-hourly for 14 days) and continued (as $100 \mathrm{mg}$ daily orally) thereafter. After a few days the patient started to speak, albeit confabulating, and the nystagmus resolved. She had a spontaneous abortion 4 weeks later. She developed severe neuropathic pain in the lower limbs that was partially responsive to carbamazepine and amitriptyline. She was discharged to inpatient rehabilitation therapy after 3 months, remaining wheelchair bound with severe Korsakoff's amnesia, confabulation and poor attention.

\section{Case 2}

A 48-year-old HIV-positive woman who had been on antiretroviral therapy (ART) for a year was admitted to a secondary-level hospital with nausea, abdominal pain and persistent vomiting for 1 month. She had an abnormal baseline creatinine level of $117 \mu \mathrm{mol} / \mathrm{l}$, reportedly as a result of previous kidney stones. Her ART regimen consisted of lamivudine, stavudine and efavirenz. The $\mathrm{CD} 4$ count was $220 \mathrm{cells} / \mu \mathrm{L}$ and the viral load was suppressed. She was on no other medication and did not use alcohol.

On admission she was found to have lactic acidosis (lactate $8.0 \mathrm{mmol} / \mathrm{L} ; \mathrm{pH} 7.2$ ) as a complication of stavudine and was discharged on Aluvia (lopinavir/ritonavir) and efavirenz. A month later she was readmitted with ongoing abdominal pain and vomiting. She was dehydrated (creatinine $158 \mu \mathrm{mol} / \mathrm{L}$ ) with hyperlactataemia (lactate $8.9 \mathrm{mmol} / \mathrm{L}$; normal $\mathrm{pH}$ ) and was treated with IV fluids containing glucose. After 3 days she complained of dizziness and double vision. A CT scan of the brain was normal. The CSF was found to have a high protein level $(1.45 \mathrm{~g} / \mathrm{L}$; normal $<0.4 \mathrm{~g} / \mathrm{L})$ but was acellular and serologically negative for syphilis. The efavirenz was stopped and she was transferred to our institution. 

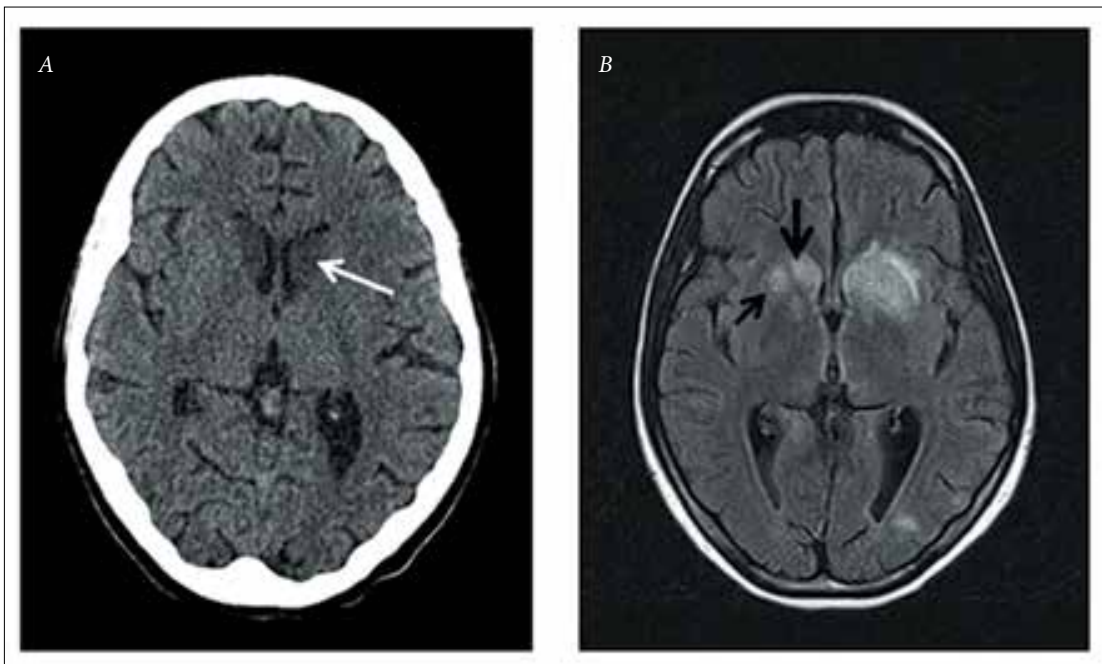

Fig. 1. (A) Case 1. CT brain scan soon after the patient presented to the secondary-level hospital, demonstrating symmetrical hypodensities in the caudate nuclei (white arrow). (B) Brain FLAIR MRI scan showing increased signal intensities in the caudate heads (large black arrow) and asymmetrical (left $>$ right) anterior lentiform nuclei (predominantly putaminal nuclei) bilaterally (small black arrow).

On admission she had tachycardia, tachypnoea and a normal blood pressure. She was confused with gaze-evoked nystagmus and bilateral abducens nerve palsies, was areflexic with mildly reduced power and limb dysdiadochokinesia, and could not sit because of severe truncal ataxia. Apart from a lactate level of $5.7 \mathrm{mmol} / \mathrm{L}$, laboratory test findings, including vitamin $\mathrm{B}_{12}$ measurement, were normal.

A clinical diagnosis of WE secondary to hyperlactaemic-induced vomiting was made. She was treated with IV thiamine $(100 \mathrm{mg}$ 8-hourly for 14 days) followed by $100 \mathrm{mg}$ orally daily. Within 24 hours the nystagmus resolved and by 14 days her eye movements were normal and the ataxia had improved to the extent that she was able to mobilise with support. At discharge she could walk independently, albeit with a broad-based gait.

\section{Case 3}

A 22-year-old man with no background illnesses had been admitted to a regional hospital intensive care unit with a rapidly deteriorating respiratory condition requiring intubation and mechanical ventilation. He was noted to have ptosis and difficulty in swallowing, and was treated with IV immunoglobulin and prednisone. Very little recovery occurred over the next 2 months and he was transferred to our institution with a tracheostomy and a percutaneous endoscopic gastrostomy (PEG) in situ, although he had been successfully weaned off ventilatory support.

We found him to be alert but emaciated. He had severe spastic anarthria without any volitional movement of the bulbar musculature, but was able to communicate in writing. It was discovered that he had been a substance abuser (daily marijuana and Mandrax) for several months, but had not used alcohol. $\mathrm{He}$ had remained well despite neglecting his diet until he developed a gastrointestinal illness characterised by severe vomiting and diarrhoea the week before admission. He then developed relatively acute difficulties with his gait and breathing, as well as bulbar symptoms. Examination showed the following striking findings: non-fatigable ophthalmoplegia and bilateral ptosis, and severe pseudobulbar palsy with a profoundly brisk jaw (and head) jerk. He was unable to open his mouth or move his tongue. The limbs were areflexic but with normal power. Findings on sensory examination were normal. His gait was mildly ataxic, but he reported that it had improved.

The results of laboratory investigations were normal, including thyroid and liver function tests and CSF examination. HIV and syphilis serology were negative. A brain MRI scan was normal.

Based on the presentation comprising spastic pseudobulbar palsy, ophthalmoplegia and ataxia following a period of profuse vomiting on a background of malnutrition, the patient was treated for WE with IV thiamine (100 mg 8-hourly for 7 days) and then $100 \mathrm{mg}$ daily orally. $\mathrm{He}$ responded within days with resolution of the eye signs and recovery of bulbar function. The PEG was removed within a month. He made a full recovery, stopped all substance use and is living a productive life.

\section{Discussion}

We describe cases of WE due to persistent vomiting but without alcoholism: hyperemesis gravidarum, drug-induced hyperlactataemia, and an acute gastrointestinal illness in a malnourished individual. Our cases highlight the importance of recognising WE when undernutrition is compounded by vomiting. ${ }^{[1]}$

WE requires IV thiamine, and treatment delay can result in significant morbidity. ${ }^{[1]}$ Thiamine is a cofactor for key enzymes involved in brain energy production such as the Krebs cycle. Brain structures such as the periaqueductal nuclei, mamillary bodies and thalami with high metabolic requirements are especially vulnerable to thiamine deficiency ${ }^{[2]}$ In non-alcoholic WE, the cerebellar vermis, midbrain reticular formation and caudate nuclei are frequently involved. ${ }^{[1]}$ Although neuroimaging often reflects signal changes in these structures, an MRI scan may be normal. ${ }^{[1]}$

The clinical diagnosis of WE can be confirmed by measuring thiamine levels using high-performance liquid chromatography. ${ }^{[1]}$ However, the diagnosis is dependent on clinical awareness and increases dramatically (to $85 \%$ ) in the presence of two of the following features: dietary deficiencies, any eye signs, cerebellar signs, and either mild memory impairment or altered mental status. ${ }^{[1]}$ There is poor consensus on the optimal IV thiamine dose, although all agree that oral treatment is ineffective. Guidelines conclude that administering thiamine intramuscularly (as the package insert suggests) is painful and that IV thiamine is safe. They recommend IV infusions of $200-500 \mathrm{mg}$ thiamine diluted in $100 \mathrm{ml}$ normal saline or $5 \%$ glucose, given over 30 minutes three times a day for 3 days, followed by $200 \mathrm{mg}$ three times daily until the patient has improved substantially before reverting to $100 \mathrm{mg}$ daily orally. ${ }^{[1,4]}$ Importantly, all high-risk individuals presenting to the emergency room should receive $200 \mathrm{mg}$ parenteral thiamine before any administration of glucose. ${ }^{[1]}$

Acknowledgements. We thank our colleagues in the Division of Neurology at Groote Schuur Hospital who assisted with the care of these patients: Christine Albertyn, Kathleen Bateman, Alan Bryer, Eddy LeePan, Alan Stanley and Lawrence Tucker.

\footnotetext{
1. Galvin R, Brathen G, Ivashynka A, Hillbom M, Tanasescu R, Leone MA, EFNS. EFNS guidelines for diagnosis, therapy and prevention of Wernicke encephalopathy. Eur J Neurol 2010;17(12):1408-1418 [http://dx.doi.org/10.1016/j.annemergmed.2007.02.007]

2. Donnino MW, Vega J, Miller J, Walsh M. Myths and misconception 2. Donnino MW, Vega J, Miller J, Walsh M. Myths and misconceptions
of Wernickés encephalopathy: What every emergency physician should know. Ann Emerg Med 2007;50(6):715-721. [http://dx.doi org/10.1111/j.1468-1331.2010.03153.x]

3. Ganie NS, Janse van Rensburg E. Wernicke's encephalopathy as a complication of gastroparesis after emergency partial antrectomy. Afr Med J 2015;105(2):157. [http://dx.doi.org/10.7196/SAMJ.9271 4. Sechi G, Serra A. Wernickés encephalopathy: New clinical settings and recent advances in diagnosis and management. Lancet Neurol 2007;6(5):442-455. [http://dx.doi.org/10.1016/ S1474-4422(07)70104-7]
}

Accepted 18 December 2015. 